\title{
COHERENT STATES AND GEODESICS: CUT LOCUS AND CONJUGATE LOCUS
}

\author{
Stefan Berceanu \\ Institute of Atomic Physics, Institute of Physics and Nuclear En- \\ gineering, Department of Theoretical Physics, P. O. Box MG-6, \\ Bucharest-Magurele, Romania; E-mail address: Berceanu@Roifa.Bitnet; \\ Berceanu@Roifa.IFA.Ro
}

\begin{abstract}
The intimate relationship between coherent states and geodesics is pointed out. For homogenous manifolds on which the exponential from the Lie algebra to the Lie group equals the geodesic exponential, and in particular for symmetric spaces, it is proved that the cut locus of the point 0 is equal to the set of coherent vectors orthogonal to $\mid 0>$. A simple method to calculate the conjugate locus in Hermitian symmetric spaces with significance in the coherent state approach is presented. The results are illustrated on the complex Grassmann manifold.
\end{abstract}

PACS numbers: 02.40.Ma; 03.65-w 


\section{INTRODUCTION}

The coherent states ${ }^{1}$ are an excellent interplay of classical and quantum mechanics. ${ }^{2}$ The local construction of Perelomov's homogeneous coherent states ${ }^{3}$ was globalized, including the Kählerian non-homogeneous manifolds. ${ }^{4}$ Simultaneously, the geometric quantization program ${ }^{5}$ furnishes, at least in principle, a tool towards the quantization program of Dirac on differentiable manifolds. Actually, using both the same mathematical objects from complex geometry, ${ }^{6}$ fibre bundles,${ }^{7}$ algebraic topology, ${ }^{8}$..., the coherent state approach and the geometric quantization are deeply related. In fact, the coherent state approach offers a straightforward recipe for geometric quantization. ${ }^{9}$

Interesting problems in both these already classical fields have not been yet attacked, however. One of them is the relationship between coherent states and geodesics.

The starting point of this paper is the Remark 3 in Ref. 10 which expresses in the language of coherent states the property, here called condition $A$ ), that for symmetric spaces the geodesics emanating from the point $o$ of the symmetric spaces are given by the exponential exp from the Lie algebra to the Lie group and all the geodesics are obtained in such a way. The aim of this report is to explore farther out this relationship.

Firstly, let us remember some notions related to geodesics. Let us fix a point $p$ of a complete Riemannian manifold $V$ and a geodesic $\gamma$ emanating from $p$. Then the cut point ${ }^{11}$ of $p$ along $\gamma$ is the first point on $\gamma$ such that, for any point $r$ beyond $q$ on $\gamma$, there is a shorter geodesic from $p$ to $r$ different from $\gamma$. A point $q$ is a conjugate point of $p$ along $\gamma$ if there is a 1-parameter family of geodesics from $p$ to $q$ neighbouring $\gamma$. Equivalent and precise definitions are given in Section 3. Here we only stress that the importance of the cut loci lies in the fact they inherit topological properties of the manifold $V . V$ may be obtained from $\mathbf{C L}_{p}$ by attaching a $n$-dimensional cell via the map Exp : $C L_{p} \rightarrow \mathbf{C L}_{p}$ and $\mathbf{C L}_{p}$ is a strong deformation retract of $V \backslash\{p\}$, where $C L$ (CL) denotes the tangent cut locus (resp. the cut locus).

In this paper it is found out that for some homogeneous manifolds there is an inti-

mate connection between the cut locus $\mathbf{C L}_{0}$ of a point on the manifold $\widetilde{\mathbf{M}}$ corresponding to a fixed coherent vector, say $\mid 0>$, and the polar divisor $\Sigma_{0}$, i.e. the locus of coherent vectors orthogonal to $\mid 0>$. The equality

$$
\mathbf{C L}_{0}=\Sigma_{0}
$$

is proved under a technical condition for the manifold, called condition $B$ ). It is stressed that condition $B$ ) imply condition $A$ ) and the well known case of Riemannian symmetric spaces $^{13-15}$ is contained as a particular case. Despite the fact that the equality (1.1) is proved only for manifolds which verify condition $B$ ), this remark is attractive even from pure mathematical point of view, due to the lack of methods to characterise the cut locus as an object of global differential geometry. ${ }^{16}$ In this paper we illustrate the results on the case of the complex Grassmann manifold $G_{n}\left(\mathbb{C}^{m+n}\right)$. The cut locus on $G_{n}\left(\mathbb{C}^{m+n}\right)$ is well known. ${ }^{13,15}$

Another contribution of this article is contained in Theorem 1 which proposes a calculation with significance in the coherent state approach of the conjugate locus for Hermitian symmetric spaces. The connection with the coherent state approach consists in the fact that the parameters $Z$ and $B$ which appear in the geodesic exponential map $Z=Z(B)$ are two different parameters of the coherent states for symmetric spaces. We also illustrate the method on the case of the complex Grassmann manifold $G_{n}\left(\mathbb{C}^{m+n}\right)$. 
However, the situation in this case is more complicated than in the case of the cut locus. In fact, there are two main contributions in this field. Wong ${ }^{14}$ has announced the expression of the conjugate locus in the Grassmann manifold, while the calculation of the tangent conjugate locus of Sakai ${ }^{15}$ shows that Wong's result is incomplete. All these problems are largely discussed elsewhere, ${ }^{17}$ where another proof of the result of Sakai on the tangent conjugate locus is given and a calculation of the conjugate locus in $G_{n}\left(\mathbb{C}^{m+n}\right)$ using Theorem 1 is also given. The only new observation ${ }^{17}$ is a geometrical characterisation of the part of the conjugate locus not found by Wong ${ }^{14,15}$ as consisting of those points of the $G_{n}\left(\mathbb{C}^{m+n}\right)$ which have at least two of the stationary angles ${ }^{18}$ with a fixed $n$-plane equal. We have included in Sec. IV only the notions necessary to illustrate the results of this paper on the example of $G_{n}\left(\mathbb{C}^{m+n}\right)$.

Some of the results included in the present work have been already briefly announced as part of a trial to find a geometrical characterisation of Perelomov's construction of coherent state manifold as Kählerian embedding into a projective space. ${ }^{19,20}$

The paper is organised as follows. In Section 2 the notation on coherent state manifolds is fixed. The result $\mathbf{C L}_{0}=\Sigma_{0}$ and some results on coherent states and conjugate points are proved in Section 3 for manifolds $\widetilde{\mathbf{M}}$ which verify condition $B$ ). Section 4 deals with the Grassmann manifold.

\section{THE COHERENT STATE MANIFOLD AND THE COHERENT VEC- TOR MANIFOLD}

Firstly we fix the notation referring to the coherent state manifold.

1. Let us consider a quantum system with symmetry, i.e. a triplet $(\mathbf{K}, G, \pi)$, where $\pi$ is an unitary irreducible representation of the Lie group $G$ on the Hilbert space $\mathbf{K}$. Let us consider the orbit

$$
\widetilde{\mathbf{M}}=\left\{\widetilde{\pi}(g)\left|\widetilde{\psi}_{0}>\right| g \in G\right\},
$$

where $\tilde{\pi}$ is the projective representation of $G$ induced by $\pi, \mid \psi_{0}>\in \mathbf{K}$ is fixed and $\xi: \mathbf{K} \rightarrow \mathbf{P K}$ is the projection $\xi(\mid \psi>) \equiv \mid \widetilde{\psi}>=\left\{e^{i \varphi}|\psi>| \varphi \in \mathbb{R}\right\}$. Then we have the bijection $\tilde{\xi}: G / K \rightarrow \widetilde{\mathbf{M}}, \tilde{\xi}(g K)=\widetilde{\pi}(g) \mid \widetilde{\psi}_{0}>$, where $K$ is the stationary group of the state $\left|\widetilde{\psi}_{0}\right\rangle$. The quantum mechanics can be realised as the elementary $G$-space ${ }^{21}$ $\left(\mathbf{P K}, \omega_{F S}, \rho^{\prime}\right)$, where $\omega_{F S}$ is the Fubini-Study (Kähler) fundamental two-form on the projective space $\mathbf{P K}$, and $\rho^{\prime}$ is the isomorphism of the Lie algebra $\mathfrak{g}$ of $G$ into the algebra of smooth functions on PK.

The keystone in the coherent state approach is to find a Hilbert space $\mathbf{L}$ and an Kählerian embedding $\iota: \widetilde{\mathbf{M}} \hookrightarrow \mathbf{P L} .^{19,22}$ Then $\widetilde{\mathbf{M}}$ is called coherent state manifold and $(\widetilde{\mathbf{M}}, \omega, \rho)$ is a hamiltonian $G$-space, with $\omega=\omega_{F S \mid \widetilde{\mathbf{M}}}=\iota^{*} \omega_{F S}, \rho=\rho_{\mid \widetilde{\mathbf{M}}}^{\prime}$. Dequantization means passing on from the dynamical system problem in the initial Hilbert space $\mathbf{K}$ to the corresponding one on $\widetilde{\mathbf{M}}$.

If $\left|\widetilde{\psi}_{0}>\equiv\right| j>$, i.e. an (anti-)dominant weight vector for compact connected simply connected Lie groups, then $\iota$ is indeed a Kählerian embedding ${ }^{21}$ and $\widetilde{M}$ coincides with the coadjoint orbit in $\mathfrak{g}^{*}$ through the root $j$ corresponding to the (anti-)dominant weight vector. ${ }^{23}$ So, furnishing both the representation $\pi=\pi_{j}$ and the Hilbert space $\mathbf{K}_{\mathbf{j}}$ of holomorphic sections with base $\widetilde{\mathbf{M}}$, it is found out that $\mathbf{L}=\mathbf{K}_{j}^{*}$, and the Borel-WeilBott theorem solves the requantization problem. ${ }^{9,24}$ Here $E^{*}$ denotes the dual of the vectorial space $E$, i.e. the space of linear functionals on $E$. 
Now we briefly discuss the embedding $\iota$ for compact complex manifolds $\widetilde{\mathbf{M}}$. In this case, the condition for the existence of the embedding $\iota$ is equivalent with the requirement for the manifold to be Hodge, ${ }^{8}$ which is the same condition as prequantization in geometric quantization. ${ }^{25}$ For example, in order to have the condition $\omega \in H^{2}(\widetilde{\mathbf{M}}, \mathbb{Z})$ fullfiled, for Hermitian symmetric spaces it is sufficient that a theorem due to HarishChandra ${ }^{10,5,26}$ to be satisfied. This theorem in the compact case is just the Borel-WeilBott theorem. The Kodaira vanishing theorem replaces the Borel-Weil-Bott theorem, as was already remarked ${ }^{27}$ in the context of cohernt states. Let now $\xi_{0}: \mathbf{M}^{\prime} \rightarrow \widetilde{\mathbf{M}}$ be a holomorphic line bundle. Another way to express the condition to have the embedding is that the line bundle $\mathbf{M}^{\prime}$ be a positive one, or, equivalently, to be ample (see Thm. 5.1. p. 89 in Ref. 28). The last condition means that there exists an integer $m_{0}$ such that for $m \geq m_{0}, M \equiv M^{\prime m}=\iota^{*}[1]$. We use the notation $[r]=H^{r}, r \in \mathbb{Z}$, where $H$ is the hyperplane bundle over $\mathbf{P L}$ and $E^{m}$ is the $m$-times tensor product of the bundle $E$ with itself. Here $\xi_{0}$ is the positive line bundle appearing in the Kodaira embedding theorem, and the embedding $\iota: \widetilde{\mathbf{M}} \hookrightarrow \mathbf{P L}=\mathbb{C P}^{N-1}$ is $^{29}$

$$
\iota \equiv \iota_{\mathbf{M}}: x \rightarrow \iota_{\mathbf{M}}(x)=\left[s_{1}(x), \ldots, s_{N}(x)\right]
$$

The line bundle $\mathbf{M}$ is furnished by the coherent state approach and is called coherent vector manifold. ${ }^{30}$ As a consequence of the Kodaira embedding theorem, the Kodaira vanishing ${ }^{28}$ theorem implies that in the sum giving the generalised Euler-Poincaré characteristic, ${ }^{8}$ only the zero term is present, and the dimension of the representation $\pi_{j}$ is furnished by the Riemann-Roch-Hirzebruch theorem (cf. Thm. 18.2.2 p. 140 in Ref. 8). There are situations in which the coherent state approach permits rapid and explicit statements, for example, for flag manifolds, the minimal exponent $N$ appearing in the Kodaira embedding theorem, $\widetilde{\mathbf{M}} \hookrightarrow \mathbf{C P}^{N-1}$, is equal with the Euler-Poincaré characteristic, $N=\chi(\widetilde{\mathbf{M}}) \cdot{ }^{19,30}$

The noncompact case is treated similarly by Kobayashi, ${ }^{31}$ the Hilbert space $\mathbf{L}$ being infinite dimensional. In the construction of Kobayashi, $\mathbf{L}$ is the dual of the Hilbert space of square integrable holomorphic $n$-forms in $\widetilde{\mathbf{M}}$. If $K$ is the kernel $2 n$-form on $\widetilde{\mathbf{M}} \times \overline{\widetilde{\mathbf{M}}}$, then the Kähler metric used by Kobayashi is $d s^{2}=\sum \partial^{2} \log K^{*} / \partial z_{i} \partial \bar{z}_{j}$, where $K(z, \bar{z})=K^{*}(z, \bar{z}) d z_{1} \wedge \ldots \wedge d z_{n} \wedge d \bar{z}_{1} \wedge \ldots \wedge d \bar{z}_{n}$.

The condition A1) (A2), respectively A3)) in Kobayashi corresponds to the condition of the set of divisors without base points (the differential of $\iota$ do not has degenerate points, respectively, the condition A1) plus the injectivity condition in the book of Griffith and Harris $\left.{ }^{29}\right)$. We remember that A1) implies that $\omega_{\mid \widetilde{\mathbf{M}}}=\iota^{*} \omega_{F S}$, while A2) and A3) implies that the application $\iota$ is a Kählerian embedding.

Now we discuss other cases in which the representation $\pi$ can be constructed. The condition to have holomorphic discrete series on homogeneous bounded symmetric domains (non-compact Hermitian symmetric spaces) results from the quoted theorem of Harish-Chandra and, more generally, the condition to have discrete series for connected semisimple Lie groups is that $\operatorname{rank} G=\operatorname{rank} K{ }^{26}$ The problem of structure of homogeneous Kähler manifolds in the context of fundamental conjecture has began to be handled in connection with the coherent states, especially for the unimodular groups. ${ }^{32}$

2 . In this section we restrict ourselves to coherent state manifolds of flag type, i.e. $\widetilde{\mathbf{M}} \approx G / K \approx G^{\mathbb{C}} / P$, where $G$ is a compact connected simply connected semisimple Lie group, $G^{\mathbb{C}}$ is the complexification of $G$ and $P$ is a parabolic subgroup of $G^{\mathbb{C}} \cdot{ }^{10}$ 
The noncompact case is handled similarly, whenever the conditions of the existence of the representation $\pi_{j}$ are fulfilled.

Let $W(G)=N(T) / C(T)$ denote the Weyl group associated with $G$, where $N(T)$ $(C(T))$ is the normalizer (the centralizer) of the Cartan group T. Let $\Sigma \subset N(T)$ be a set of elements such that quotient space $W(G) / W(K)$ is made of the coset classes $\{s C(T)\} W(K), s \in \Sigma$. Then there is an open covering of $\widetilde{\mathbf{M}}$ by $\left(\mathcal{V}_{s}\right)_{s \in \Sigma}$, where $\mathcal{V}_{s}=\pi_{j}(s) \mathcal{V}_{0}, s \in \Sigma .^{30}$ The coherent state vectors corresponding to the points of the neighbourhood $\mathcal{V}_{0} \subset \widetilde{\mathbf{M}}$ around $Z=0$ are

$$
\left|Z, j>=\exp \sum_{\varphi \in \Delta_{n}^{+}}\left(Z_{\varphi} F_{\varphi}^{+}\right)\right| j>,|\underline{Z}>=<Z| Z>^{-1 / 2} \mid Z>\in \mathbf{M}
$$

where $Z \in \mathbb{C}^{n}$ are local coordinates and $n$ is the dimension of the manifold $\widetilde{\mathbf{M}}$. Here

$$
F_{\varphi}^{ \pm}=\pi^{* \prime}\left(f_{\varphi}^{ \pm}\right)
$$

$\pi^{\prime}=d \pi, \pi^{\prime}$ is the isomorphism of the Lie algebra $\mathfrak{g}$ of $G$ onto the Lie algebra of operators on $\mathbf{K}, \pi^{*}$ is the group isomorphism $G^{\mathbb{C}} \rightarrow \pi^{*}\left(G^{\mathbb{C}}\right)$,

$$
\pi^{*}\left(e^{Z}\right)=\exp \left(\pi^{* \prime}(Z)\right), Z \in \mathfrak{g}^{\mathbb{C}},
$$

$\pi^{* \prime}\left(\mathfrak{g}^{\mathbb{C}}\right)$ is the complexification of the Lie algebra $\pi^{\prime}(\mathfrak{g})$, the subindex $n(c)$ abbreviates the noncompact (respectively, compact), $\Delta$ are the roots and $\Delta^{+}$the positive roots.

We also use the notation

$$
f_{\varphi}^{ \pm}= \begin{cases}k_{\varphi}^{ \pm}=i e_{ \pm \varphi}, & \text { for } X_{n}, \\ e_{\varphi}^{ \pm}=e_{ \pm \varphi}, & \text { for } X_{c} .\end{cases}
$$

where $e_{\varphi}^{ \pm}=e_{ \pm \varphi}$ are the part of the Cartan-Weyl base corresponding to $\mathfrak{m}$. Here $\mathfrak{g}=\mathfrak{k} \oplus \mathfrak{m}$ is the Cartan decomposition of the Lie algebra $\mathfrak{g}$ of $\mathbf{G}$ and $\mathfrak{k}$ is the Lie algebra of $\mathbf{K}$.

The homogeneous symmetric spaces are obtained as

$$
X_{n, c}=\exp \sum_{\varphi \in \Delta_{n}^{+}}\left(B_{\varphi} f_{\varphi}^{+}-\bar{B}_{\varphi} f_{\varphi}^{-}\right) \cdot o
$$

where $o=\lambda(e), e$ is the unit element in $G$ and $\lambda$ is the canonical projection $\lambda: G \rightarrow$ $G / K$. Let also the notation

$$
\begin{gathered}
\left|B, j>=\exp \sum_{\varphi \in \Delta_{n}^{+}}\left(B_{\varphi} F_{\varphi}^{+}-\bar{B}_{\varphi} F_{\varphi}^{-}\right)\right| j>, \\
|B, j>\equiv| \underline{Z, j}>.
\end{gathered}
$$

Note that

$$
F_{\varphi}^{+}\left|j>\neq 0, F_{\varphi}^{-}\right| j>=0, H_{i}\left|j>=j_{i}\right| j>,
$$

where $\varphi \in \Delta_{n}^{+}, H_{i}=\pi^{*}\left(h_{i}\right),\left\{h_{i}\right\}$ is a base of the Cartan subalgebra and $i=$ $1, \ldots, \operatorname{rank} G$. 
3. We now state more precisely the definition of the coherent vector manifold $\mathbf{M}$ corresponding to flag manifolds $\widetilde{\mathbf{M}}$. Let $\mathbf{M}^{\prime}$ be the holomorphic line bundle $\mathbf{M}^{\prime}=$ $\xi_{0}^{-1}(\widetilde{\mathbf{M}}) \rightarrow G_{c} / P$ associated by the holomorphic character $\chi=\chi_{j}$ of $P$ to the principal bundle $P \rightarrow G^{\mathbb{C}} \rightarrow G^{\mathbb{C}} / P$, i.e. the line bundle obtained identifying $(g, \chi(p) w)$ with $(g p, w)$, where $p \in P, w \in \mathbb{C}$.

In fact, if $\left(\mathbf{M}^{\prime}, \omega, J\right)$ is the compact Kähler manifold ( $J$ is the complex structure, $J=\operatorname{ad}(Z) \mid \mathfrak{m}$ and $Z$ is the central element of the Lie algebra $\mathfrak{k})$, then $\left(\mathbf{M}^{\prime}, \nabla, h\right)$ is a quantization bundle over $\widetilde{\mathbf{M}},{ }^{5}$ where $h$ is the hermitian form on the tautological line bundle [-1] over PL. Then, on [-1], $h$ is given by $h: z \rightarrow|z|^{2}$. Also $\operatorname{curv}(\nabla)=-2 \pi i \omega$, so $\omega \in c_{1}\left(\mathbf{M}^{\prime}\right)=[\omega]_{\text {de Rham }}$.

If $\varphi_{i}: V_{i} \times \mathbf{C} \rightarrow \xi_{0}^{-1}\left(V_{i}\right)$ is the local trivialization of the holomorphic line bundle $\mathbf{M}^{\prime} \rightarrow \widetilde{\mathbf{M}}$, then a global section is given by

$$
\mid s_{i}(m)>=\left(g_{i}\left(Z_{i}\right), f_{s_{i}}\left(Z_{i}\right)\right)=\left(g_{i}\left(Z_{i}\right),<s_{i} \mid Z_{i}>\right),
$$

where $m=g_{i}\left(Z_{i}\right) \in V_{i}$ are matrix elements determined by the local coordinates $Z_{i}$. Then the scalar product on the line bundle $\mathbf{M}^{\prime} \rightarrow \widetilde{\mathbf{M}}$ is given by ${ }^{9,30}$

$$
\begin{aligned}
<s_{i} \mid s_{i}^{\prime}> & =\int_{\widetilde{\mathbf{M}}} h_{X}\left(s_{i}(X), s_{i}^{\prime}(X)\right) \frac{\omega^{n}(X)}{n !} \\
& =<f_{s_{i}}, f_{s_{i}^{\prime}}>=\int_{\widetilde{\mathbf{M}}} h_{X}\left(f_{s_{i}}(X), f_{s_{i}^{\prime}}(X)\right) \frac{d \mu(X)}{<X \mid X>},
\end{aligned}
$$

where $d \mu(X)$ is the Haar measure on $\widetilde{\mathbf{M}} \approx G^{\mathbb{C}} / P$.

The scalar product in (2.12) is also a hermitian scalar product of sections with base $\widetilde{\mathbf{M}}$ in the $D_{\widetilde{\mathbf{M}}^{-}}$module of differentiable operators on $\widetilde{\mathbf{M}} \cdot{ }^{10}$

When both the dequantization and the requantization can be done, then the Hilbert space $\mathbf{K}_{j}$ attached to the representation $\pi_{j}$ and the initial $\mathbf{K}$ are isomorphic. ${ }^{9,27}$

\section{THE CUT LOCUS AND COHERENT STATES}

In this section we shall be concerned with various aspects of the relationship between geodesics and coherent states. We briefly review some definitions used in the Introduction.

1. Let $V$ be compact Riemannian manifold of dimension $n, p \in V$ and let $\operatorname{Exp}_{p}$ be the (geodesic) exponential map at the point $p$. Let $C_{p}$ denote the set of vectors $X \in V_{p}$ (the tangent space at $\left.p \in V\right)$ for which $\operatorname{Exp}_{p} X$ is singular. A point $q$ in $V\left(V_{p}\right)$ is conjugate to $p$ if it is in $\mathbf{C}_{p}=\operatorname{Exp} C_{p}\left(C_{p}\right)^{12}$ and $\mathbf{C}_{p}\left(C_{p}\right)$ is called the conjugate locus (resp. tangent conjugate locus) of the point $p$.

Let $q \in V$. The point $q$ is in the cut locus $\mathbf{C L}_{p}$ of $p \in V$ if it is nearest point to $p \in V$ on the geodesic joining $p$ with $q$, beyond which the geodesic ceases to minimise its arc length. ${ }^{11}$ More precisely, let $\gamma_{X}(t)=\operatorname{Exp} t X$ be a geodesic emanating from $\gamma_{X}(0)=p \in V$, where $X$ is a unit vector from the unit sphere $S_{p}$ in $V_{p} . t_{0} X$ (resp. $\left.\operatorname{Exp} t_{0} X\right)$ is called a tangential cut point (cut point) of $p$ along $t \rightarrow \operatorname{Exp} t X(0 \leq t \leq s)$ if the geodesic segment joining $\gamma_{X}(0)$ and $\gamma_{X}(t)$ is a minimal geodesic for any $s \leq t_{0}$ but not for any $s>t_{0}$.

Let us define the function $\mu: S_{p} \rightarrow \mathbb{R}^{+} \cup \infty, \mu(X)=r$, if $q=\operatorname{Exp} r X \in \mathbf{C L}_{p}$, and $\mu(X)=\infty$ if there is no cut point of $p$ along $\gamma_{X}(t)$. Setting $I_{p}=\{t X, 0<t<\mu(X)\}$, then $\mathbf{I}_{p}=\operatorname{Exp} I_{p}$ is called the interior set at $p$. Then: 
1) $\mathbf{I}_{p} \cap \mathbf{C L}_{p}=\emptyset, V=\mathbf{I}_{p} \cup \mathbf{C} \mathbf{L}_{p}$, the closure $\overline{\mathbf{I}}_{p}=V$, and $\operatorname{dim} \mathbf{C L}_{p} \leq n-1$;

2) $I_{p}$ is a maximal domain containing $0=0_{p} \in V_{p}$ on which $\operatorname{Exp}_{p}$ is a diffeomorphism and $\mathbf{I}_{p}$ is the largest open subset of $V$ on which a normal coordinate system around $p$ can be defined.

The relative position of $\mathbf{C L}_{0}$ and $\mathbf{C}_{0}$ is given by Theorem 7.1 p. 97 in Ref. 11 reproduced below.

Let the notation $\gamma_{t}=\gamma_{X}(t)$. Let $\gamma_{r}$ be the cut point of $\gamma_{0}$ along a geodesic $\gamma=\gamma_{t}, 0 \leq t<\infty$. Then, at least one (possibly both) of the following statements holds:

(1) $\gamma_{r}$ is the first conjugate point of $\gamma_{0}$ along $\gamma$;

(2) there exists, at least, two minimising geodesics from $\gamma_{0}$ to $\gamma_{r}$.

Crittenden ${ }^{33}$ has shown that for the case of simply connected symmetric spaces, the cut locus is identified to the first conjugate point. Generally, the situation is more complicated. ${ }^{34,35}$

Here are simple examples of cut loci. For the sphere $S^{n}$, the cut locus of a point reduces to the antipodal point, while the tangent cut locus $C L$ is the sphere of radius $\pi$ with centre at the origin of the tangent space. For $\mathbb{C P}^{n}, C L$ is also the sphere of radius $\pi$ with centre at the origin of the tangent space to $\mathbb{C P}^{n}$ at the given point, while CL is the hyperplane at infinity $\mathbb{C P}^{n-1}$. Except few situations, e. g. the ellipsoid, even for low dimensional manifolds as the (asymmetric Berger's spheres) $S^{3}$, CL is not known explicitly. Helgason ${ }^{12}$ has shown that the cut locus of a compact connected Lie group, endowed with a bi-invariant Riemannian metric is stratified, i.e. it is the disjoint union of smooth submanifolds of $V$. This situation will be illustrated on the case of complex Grassmann manifold. Using a geometrical method, Wong ${ }^{13,14,36}$ has studied conjugate loci and cut loci of the Grassmann manifolds emphasising also their stratification. Sakai ${ }^{37}$ has found out the cut locus of the connected compact symmetric manifold $V=U(n) / O(n)$, which has $\pi_{1}(V) \cong \mathbb{Z}$. By refining the results of Ch. VII, $\S 5$ "Control over singular set" from Helgason's book, ${ }^{12}$ Sakai ${ }^{15,38}$ studied the cut locus of a point in a compact symmetric space which is not necessarily simply connected and showed that it is determined by the cut locus of a maximal totally geodesic flat torus of $V$. Takeuchi ${ }^{39}$ has also proved the stratified structure of $\mathbf{C L}$ and $\mathbf{C}$ for compact symmetric manifolds. For other references see Kobayashi ${ }^{16}$. However, the expression of the conjugate locus as subset of the Grassmann manifold is not known explicitely. This problem is largely discussed elsewhere. ${ }^{17}$ In $\S 4$ of this paper we shall only collect the main results on this problem.

Most considerations in this Section concern only manifolds with the property

$$
\left.\operatorname{Exp}\right|_{o}=\lambda \circ \exp \mid \mathfrak{m} .
$$

Here $\mathfrak{g}=\mathfrak{k} \oplus \mathfrak{m}$ is the orthogonal decomposition with respect to the $B$-form as explained below at $B$ ), $\operatorname{Exp}_{p}: \widetilde{\mathbf{M}}_{p} \rightarrow \widetilde{\mathbf{M}}$ is the geodesic exponential map (cf. Ref. 12 p. 33) and $\exp : \mathfrak{g} \rightarrow G$.

In fact, $A$ ) expresses that the geodesics in $\widetilde{\mathbf{M}}$ are images of one-parameter subgroups of $\widetilde{\mathbf{M}} \approx G / K$. The symmetric spaces have property $A$ ) (cf. Thm. 3.3 p. 208 in Ref. 12).

We shall also be concerned with manifolds $\widetilde{\mathbf{M}}$ verifying the following condition: 
$B)$ On the Lie algebra $\mathfrak{g}$ of $G$ there exists an $A d(G)$-invariant, symmetric, nondegenerate bilinear form $B$ such that the restriction of $B$ to the Lie algebra $\mathfrak{k}$ of $K$ is likewise non-degenerate.

We point out that if the homogeneous space $\widetilde{\mathbf{M}} \approx G / K$ verifies $B)$, then it also verifies $A$ ) (cf. Corollary 2.5, Thm. 3.5 and Corollary 3.6 Chapter $\mathrm{X}$ in Ref. 11). Indeed, if $\mathfrak{g}=\mathfrak{k} \oplus \mathbf{m}$ is the orthogonal decomposition relative to the $B$-form on $\mathfrak{g}$, then $\mathfrak{m}$ is canonically identified with the tangent space at $o, \widetilde{\mathbf{M}}_{o} . \quad B$ ) implies a (possibly indefinite) $G$-invariant metric on $\widetilde{\mathbf{M}}$. It follows that $G / K$ is reductive, i.e. $[\mathfrak{k}, \mathfrak{k}] \subset \mathfrak{k}$ and $[\mathfrak{k}, \mathfrak{m}] \subset \mathfrak{m}$. If $B$ ) is true, then $\widetilde{\mathbf{M}}$ is naturally reductive (see p. 202 in Ref. 11) and $A$ ) is also verified. The symmetric spaces verify besides the conditions of reductive spaces, the condition $[\mathfrak{m}, \mathfrak{m}] \subset \mathfrak{k}$ and, of course, $A$ ) is verified too (see Thm. 3.2 Ch. Xl in Ref. 11).

Thimm ${ }^{40}$ furnishes as another examples of homogeneous spaces verifying $B$ ), besides the symmetric spaces, the Lie groups with bi-invariant metric and the normal homogeneous spaces (i.e. $B$ is positive definite). Kowalski ${ }^{41}$ studied generalised symmetric spaces still verifying condition $A$ ). See also Montgomery. ${ }^{42}$

2. Now we remember that in Ref. 10 we did the following Remark, which is in fact E. Cartan's theorem (see e.g. Thm. 3.3 p. 208 in Ref. 12) on geodesics on symmetric spaces expressed in the coherent state setting:

Remark 1 The vector $\left|t B, j>=\exp \pi_{j}^{* \prime}(t B)\right| j>\in \mathbf{M}, B \in \mathfrak{m}$, describes trajectories in $\mathbf{M}$ corresponding to the image in the manifold of coherent states $\widetilde{\mathbf{M}} \hookrightarrow \mathbf{P L}$ of geodesics through the identity coset element on the symmetric space $X \approx G / K$. The dependence $Z(t)=Z(t B)$ appearing when one passes from eq. (2.8) to eq. (2.3) describes in $\mathcal{V}_{0}$ a geodesic.

We shall reformulate Remark 1 in a way very useful even for practical calculations. The proof presented below, true in the particular case of hermitian symmetric spaces, implies also Thm. 1.

Remark 2 For an $n$ - dimensional manifold $X \approx G / K$ which has Hermitian symmetric space structure, the parameters $B_{\varphi}$ in formula (2.8) of normalised coherent states are normal coordinates in the normal neighbourhood $\mathcal{V}_{0} \approx \mathbb{C}^{n}$ around the point $Z_{\varphi}=0$ on the manifold $X$.

Proof. The Harish-Chandra embedding theorem can be used (cf. e.g. Ref. 26; see also Ref. 10 for the present context). This theorem asserts that the map $M^{+} \times K^{\mathbb{C}} \times M^{-} \rightarrow$ $G^{\mathbb{C}}$ given by $\left(m^{+}, k, m^{-}\right) \rightarrow m^{+} k^{-}$is a complex analytic diffeomorphism onto an open dense subset of $G^{\mathbb{C}}$ that contains $G_{n}$. Let $\mathfrak{m}^{ \pm}$be the $\pm i$ eigenspaces of $J$ and $M^{ \pm}$ the (unipotent, Abelian) subgroups of $G^{\mathbb{C}}$ corresponding to $\mathfrak{m}^{ \pm}$. Then, in particular, $b: \mathfrak{m}^{+} \rightarrow X_{c}=G^{\mathbb{C}} / P, b(X)=\exp (X) P$ is a complex analytic diffeomorphism of $\mathfrak{m}^{+}$onto a dense subset of $X_{c}$ (that contains $X_{n}$ ) and the Remark follows because the requirement $A$ ) is fulfilled for the symmetric spaces.

The proof of Remark 2 also shows that the dependence $Z=Z(B)$, with $B \in \mathfrak{m}^{+}$, and $Z$ parametrizing $\widetilde{\mathbf{M}}$, obtained passing from eq. (2.8) to (2.3) using the relations (2.10) (the Baker-Campbell-Hausdorff formulas), ${ }^{10}$ expresses in fact the geodesic exponential $\operatorname{Exp}_{0}: \widetilde{\mathbf{M}}_{0} \rightarrow \widetilde{\mathbf{M}}$. So we have proved the following 
Theorem 1 Let $\widetilde{\mathbf{M}}$ be a coherent state manifold with Hermitian symmetric space structure, parametrized in $\mathcal{V}_{0}$ around $Z=0$ as in eqs. (2.3), (2.8). Then the conjugate locus of the point $o$ is obtained vanishing the Jacobian of the exponential map $Z=Z(B)$ and the corresponding transformations of the chart from $\mathcal{V}_{0}$.

The situation is very transparent in the case of the complex Grassmann manifold $X_{c}=G_{n}\left(\mathbb{C}^{n+m}\right)=S U(n+m) / S(U(n) \times U(m))$ and his noncompact dual $X_{n}=$ $S U(n, m) / S(U(n) \times U(m))$. There ${ }^{10}$

$$
\begin{aligned}
X_{n, c} & =\exp \left(\begin{array}{cc}
0 & B \\
\pm B^{*} & 0
\end{array}\right) o=\left(\begin{array}{cc}
\operatorname{co} \sqrt{B B^{*}} & B \frac{\operatorname{si} \sqrt{B^{*} B}}{\sqrt{B^{*} B}} \\
\pm \frac{\operatorname{si} \sqrt{B^{*} B}}{\sqrt{B^{*} B}} B^{*} & \operatorname{co} \sqrt{B^{*} B}
\end{array}\right) o \\
& =\left(\begin{array}{cc}
\mathbb{1} & Z \\
0 & \mathbb{1}
\end{array}\right)\left(\begin{array}{cc}
\left(\mathbb{1} \mp Z Z^{*}\right)^{1 / 2} & 0 \\
0 & \left(\mathbb{1} \mp Z^{*} Z\right)^{1 / 2}
\end{array}\right)\left(\begin{array}{cc}
\mathbb{1} & 0 \\
\pm Z^{*} & \mathbb{1}
\end{array}\right) o \\
& =\exp \left(\begin{array}{ll}
0 & Z \\
0 & 0
\end{array}\right) P,
\end{aligned}
$$

where $B^{*}$ denotes the hermitian conjugate of the matrix $B$. co is an abbreviation for the circular cosine cos (resp. the hyperbolic cosine coh) for $X_{c}$ (resp. $X_{n}$ ) and similarly for si. The $-(+)$ sign in the equation above corresponds to the compact (resp. noncompact) $X$.

Here $Z$ and $B$ are $n \times m$ matrices related by the relation

$$
Z=B \frac{\operatorname{ta} \sqrt{B^{*} B}}{\sqrt{B^{*} B}}
$$

and ta is an abbreviation for the hyperbolic tangent tgh (resp. the circular tangent tg) for $X_{n}$ (resp. $\left.X_{c}\right)$. The dependence $Z=Z(B)$ describes in fact Exp : $G_{n}\left(\mathbb{C}^{n+m}\right)_{e} \rightarrow$ $G_{n}\left(\mathbb{C}^{n+m}\right)$ in $\mathcal{V}_{0}$. Indeed, the equation of geodesics for $X_{c, n}$ is ${ }^{17}$

$$
\frac{d^{2} Z}{d t^{2}}-2 \epsilon \frac{d Z}{d t} Z^{+}\left(\mathbb{1}+\epsilon Z Z^{+}\right)^{-1} \frac{d Z}{d t}=0,
$$

where $\epsilon=1(-1)$ for $X_{c}$ (resp. $X_{n}$ ). It is easy to see that (3.2) verifies (3.3) with the initial condition $\dot{Z}(0)=B$.

$Z$ and $B$ in the eq. (3.2) of geodesics are in the same time the parameters describing the coherent states in the paramerization given by eq. (2.3) and respectively (2.8).

3. Firstly, let us introduce a notation for the polar divisor of $\mid 0>\in \mathbf{M}$ :

$$
\Sigma_{0}=\{|\psi>||\psi>\in \mathbf{M},<0| \psi>=0\} .
$$

This denomination is inspired from that one used by $\mathrm{Wu}^{43}$ in the case of the Grassmann manifold.

We shall prove the following 
Theorem 2 Let $\widetilde{\mathbf{M}}$ be a homogeneous manifold $\widetilde{\mathbf{M}} \approx G / K$. Suppose that there exists an unitary irreducible representation $\pi_{j}$ of $G$ such that in a neighbourhood $\mathcal{V}_{0}$ around $Z=0$ the coherent states are parametrized as in eq. (2.3). Then the manifold $\widetilde{\mathbf{M}}$ can be represented as the disjoint union

$$
\widetilde{\mathbf{M}}=\mathcal{V}_{0} \cup \Sigma_{0}
$$

Moreover, if the condition B) is true, then

$$
\Sigma_{0}=\mathbf{C L}_{0}
$$

and

$$
\mathcal{V}_{0}=\mathbf{I}_{0}
$$

Proof. We can take $|\psi\rangle=\mid \psi(Z)>\in \mathbf{M}$ such that the parameters $Z$ are in $\mathbb{C}^{n}$ as in formula (2.3). Now, the second relation (2.10) implies that $\langle 0 \mid \psi\rangle=1$ for $\mid \psi>\in \xi_{0}^{-1}\left(\mathcal{V}_{0}\right)$. It follows that the equation

$$
\cos \theta=0
$$

where

$$
\cos \theta=\frac{|<0| \psi>\mid}{\|0\|^{1 / 2}\|\psi\|^{1 / 2}}=\|\psi\|^{-1 / 2}
$$

does not have solutions for $\mid \psi>\in \xi_{0}^{-1}\left(\mathcal{V}_{0}\right)$, and the representation (3.4) follows.

To prove relation (3.5) if $B$ ) is true, use is made of Thm. 7.4 and the subsequent remark at p. 100 from Ref. 11, reproduced at the beginning of this section in an enriched version. The theorem essentially says that any Riemannian manifold $\widetilde{\mathbf{M}}$ is the disjoint union of the cut locus (closed cell) and the largest open cell of $\widetilde{\mathbf{M}}$ on which normal coordinates can be defined. But $Z \in \mathbb{C}^{n}$ for points of $\mathcal{V}_{0}$ corresponding to the largest normal coordinates $B \in \mathfrak{m}$, because $B$ ) implies $A$ ).

Farther we shall prove a Corollary of Thm. 2. This is related to the angle $\theta$ appearing in eq. (3.9).

Firstly, let us introduce the (hermitian elliptic) Cayley distance ${ }^{44}$ in the projective space. Let $(\cdot, \cdot)$ be the scalar product in $\mathbf{K}$. If $\xi: \mathbf{K} \backslash\{0\} \rightarrow \mathbf{P K}$ is the natural projection $\xi: \omega \rightarrow[\omega]$, then the Cayley distance is

$$
d_{c}\left(\left[\omega^{\prime}\right],[\omega]\right)=\arccos \frac{\left|\left(\omega^{\prime}, \omega\right)\right|}{\left\|\omega^{\prime}\right\|\|\omega\|} .
$$

The infinite dimensional case is argued in Ref. 31. Before proving the Corollary, we shall present 
Remark 3 (Geometrical significance of transition amplitudes for coherent states) Let $\mid \underline{Z}>\in \mathbf{M}, Z \in \mathcal{V}_{0}$ as in (2.3) and $\iota: \widetilde{\mathbf{M}} \hookrightarrow \mathbf{P L}$ the embedding of the coherent state manifold into the projective space. Then the angle $\theta=\theta\left(Z, Z^{\prime}\right)$ defined by

$$
\theta \equiv \arccos \left|<\underline{Z^{\prime}}\right| \underline{Z}>\mid
$$

is equal with the geodesic distance joining $\iota(Z)$ and $\iota\left(Z^{\prime}\right)$,

$$
\theta=d_{c}\left(\iota\left(Z^{\prime}\right), \iota(Z)\right) .
$$

More generally, the (Cauchy) formula is true

$$
<\underline{Z^{\prime}} \mid \underline{Z}>=\frac{\left(\iota\left(Z^{\prime}\right), \iota(Z)\right)}{\left\|\iota\left(Z^{\prime}\right)\right\|\|\iota(Z)\|} .
$$

Proof. The relation (3.13) is an immediate consequence of the fact that the complex analytic line bundle $\mathbf{M}$ over $\widetilde{\mathbf{M}}$ is projectively induced (see p. 139 in Ref. 8), i.e. the coherent state manifold $\mathbf{M}$ is the pull-back of the hyperplane bundle $H=[1]$ on $\mathbf{P L}$, i.e. $\mathbf{M}=\iota^{*}[1]{ }^{27}$

The denomination of eq. (3.13) as the Cauchy formula is due to the fact that for the Plücker embedding of the Grassmann manifold this formula is nothing else than the (Binet-) Cauchy formula. ${ }^{45}$

Combining Remark 3 and Theorem 2, we get the following

Corollary 1 Suppose that $\widetilde{\mathbf{M}}$ is an homogeneous manifold verifying B) and admitting the embedding $\iota: \widetilde{\mathbf{M}} \hookrightarrow \mathbf{P L}$. Let $0, Z \in \widetilde{\mathbf{M}}$. Then $Z \in \mathbf{C L}_{0}$ iff the Cayley distance between the images $\iota(0), \iota(Z) \in \mathbf{P L}$ is $\pi / 2$

$$
d_{c}(\iota(0), \iota(Z))=\pi / 2 .
$$

\section{AN EXAMPLE: THE COMPLEX GRASSMANN MANIFOLD}

The results of Section 3 will be illustrated on the example of the complex Grassmann manifold. The calculation of the cut locus on $G_{n}\left(\mathbb{C}^{n+m}\right)$ was announced by Wong ${ }^{13}$ and now more proofs (see e.g. Sakai ${ }^{15}$ and also Ref. 31) are available. Also Wong ${ }^{14}$ has announced the conjugate locus on the Grassmann manifold, but, as far as I know, the proof has not been published. Even more, the results of Wong on conjugate locus on Grassmann manifold were contested by Sakai, ${ }^{15}$ who showed that the result of Wong is incomplete.

The explicit calculation of the conjugate locus in the manifold using Theorem 1 is presented elsewhere. ${ }^{17}$ Another proof of the results of Sakai referring to the tangent conjugate locus is also presented there. Here we just indicate the parameters appearing in the calculation in order to illustrate how the assertions of Section 3 referring to the cut locus and conjugate locus work in an concrete example. However, we do not have an explicit expression for the part of the conjugate locus lost by Wong and only a geometrical characterisation in terms of the stationary angles. 
1. Firstly we fix the notation concerning the geometric construction of coherent state manifold when $\widetilde{\mathbf{M}}$ is the complex Grassmann manifold (the manifold of Slater determinants ${ }^{46}$ ).

Let $\mathbf{O}$ be the $n$-plane passing through the origin of $\mathbb{C}^{N}(N=n+m)$ corresponding to $Z=0$ in $\mathcal{V}_{0} \subset G_{n}\left(\mathbb{C}^{N}\right)$ in the representation (2.3). Then $Z \in \mathcal{V}_{0} \approx \mathbb{C}^{n \times m}$ iff there are $n$ vectors $\boldsymbol{z}_{1}, \ldots, \boldsymbol{z}_{n} \in \mathbb{C}^{N}$ such that

$$
Z=z_{1} \wedge \ldots \wedge z_{n} \neq 0 .
$$

We use the Pontrjagin coordinates. Fixing the canonical basis $\boldsymbol{e}_{1}, \ldots, \boldsymbol{e}_{N}$ for $\mathbb{C}^{N}$, then

$$
\boldsymbol{z}_{i}=\boldsymbol{e}_{i}+\sum_{\alpha=n+1}^{N} Z_{i \alpha} \boldsymbol{e}_{\alpha}
$$

If the weight $j$ is taken as ${ }^{10}$

$$
j=(\underbrace{1, \ldots, 1}_{n}, \underbrace{0, \ldots, 0}_{m}),
$$

then we have the equality ${ }^{17}$ of the scalar product $<\cdot \mid \cdot>$ of coherent vectors from $\mathbf{M}$ and of the hermitian scalar product $((\cdot, \cdot))$ in the holomorphic line bundle $\operatorname{det}^{* 6}$

$$
<Z^{\prime} \mid Z>=\left(\left(\hat{Z}^{\prime}, \hat{Z}\right)\right)=\operatorname{det}\left(\left(\boldsymbol{z}^{\prime}{ }_{i}, \boldsymbol{z}_{j}\right)\right)_{i, j=1, \ldots, n}=\operatorname{det}\left(\mathbb{1}_{n}+Z Z^{\prime *}\right) .
$$

We have used the notation

$$
\hat{Z}=\left(\mathbb{1}_{n}, Z\right),
$$

where $Z$ is an $n \times m$ matrix and $\mathbb{1}_{n}$ is the unity $n \times n$ matrix.

So, the parameters $Z$ in formula (2.3) for the Grassmann manifold of coherent states are the Pontrjagin ${ }^{47}$ coordinates $Z$ in formula (??).

Let us also introduce the Plücker coordinates $Z^{i_{1} \ldots i_{n}}$, i.e.

$$
Z=\sum_{1 \leq i_{1}<\ldots<i_{n} \leq N} Z^{i_{1} \ldots i_{n}} \boldsymbol{e}_{i_{1}} \wedge \ldots \wedge \boldsymbol{e}_{i_{n}} .
$$

Let $\iota: G_{n}(\mathbf{K}) \hookrightarrow \mathbf{P L}$ be the Plücker embedding, where $\mathbf{K}=\mathbb{C}^{N}, \mathbf{L}=\mathbb{C}^{* N(m)}$, $N(m)=\left(\begin{array}{l}N \\ n\end{array}\right)-1$. Using the notation of Section 2 for $X=X_{c}=G_{n}\left(\mathbb{C}^{N}\right)$, then $\mathbf{M}^{\prime}=\mathbf{M}$, that is $m_{0}=1$ in $\mathbf{M}^{\prime m_{0}}=\mathbf{M}$, (i.e. the line bundle det* is not only ample, but very ample ${ }^{28}$ ) and $\mathbf{M}=\iota^{*}[1]$, where [1] is the hyperplane section $H$ in $\mathbf{L}$.

The (Binet-) Cauchy formula ${ }^{45}$ invoked in eq. (3.13) reads explicitly

$$
\operatorname{det}\left(\left(\boldsymbol{z}_{i}^{\prime}, \boldsymbol{z}_{j}\right)\right)_{i, j=1, \ldots, n}=\sum_{1 \leq i_{1}<\ldots<i_{n} \leq N} Z^{i_{1} \ldots i_{n}} \bar{Z}^{i_{1} \ldots i_{n}} .
$$

2. Now we fix the notation referring to the Schubert varieties.

Let the sequences of integers

$$
\begin{gathered}
\omega=\{0 \leq \omega(1) \leq \ldots \leq \omega(n) \leq m\}, \\
\sigma(i)=\omega(i)+i, i=1, \ldots, n .
\end{gathered}
$$


The Schubert varieties are defined $a^{47}$

$$
Z(\omega)=\left\{X \in G_{n}\left(\mathbb{C}^{n+m}\right) \mid \operatorname{dim}\left(X \cap \mathbb{C}^{\sigma(i)}\right) \geq i\right\} .
$$

$Z(\omega)$ are closed cells in the Grassmann manifold. The "jumps" sequence ${ }^{48}$ is introduced as

$$
I_{\omega}=\left\{i_{0}<i_{1}<\ldots<i_{l-1}<i_{l}=n\right\},
$$

where

$$
\omega\left(i_{h}\right)<\omega\left(i_{h+1}\right), \omega(i)=\omega\left(i_{h-1}\right), i_{h-1}<i \leq i_{h}, h=1, \ldots, l .
$$

Let us consider the subset of generic elements of $Z(\omega)^{47}$

$$
Z^{\prime}(\omega)=\left\{X \subset G_{n}\left(\mathbb{C}^{n+m}\right) \mid \operatorname{dim}\left(X \cap \mathbb{C}^{\sigma\left(i_{h}\right)}\right)=i_{h}, i_{h} \in I_{\omega}\right\} .
$$

The condition to get generic elements $Z$ of $Z(\omega), Z \in V_{0} \cap Z(\omega) \subset Z^{\prime}(\omega)$, is : ${ }^{47,49}$

$$
Z_{i j}=0, \quad j>\omega(i), i=1, \ldots, n .
$$

Let also the notation

$$
\begin{gathered}
V_{l}^{p}=\left\{Z \subset G_{n}\left(\mathbb{C}^{n+m}\right) \mid \operatorname{dim}\left(Z \cap \mathbb{C}^{p}\right) \geq l\right\}, \\
W_{l}^{p}=V_{l}^{p}-V_{l+1}^{p}=\left\{Z \subset G_{n}\left(\mathbb{C}^{n+m}\right) \mid \operatorname{dim}\left(Z \cap \mathbb{C}^{p}\right)=l\right\}, \\
\omega_{l}^{p}=(\underbrace{p-l, \ldots, p-l}_{l}, \underbrace{m, \ldots, m}_{n-l}) .
\end{gathered}
$$

Then $^{14,36,17}$

$$
V_{l}^{p}=Z\left(\omega_{l}^{p}\right) ; \quad W_{l}^{p}=Z^{\prime}\left(\omega_{l}^{p}\right) .
$$

3. Now we briefly remember some notions referring to the stationary angles.

Let $Z^{\prime}, Z$ be two $n$-planes of $G_{n}\left(\mathbb{C}^{n+m}\right)$ given as in eq. (4.1). Then the $(n)$ stationary angles (see Jordan ${ }^{18}$ for the real case), of which most $r=\min (m, n)$ are nonzero, are defined as the stationary angles $\theta \in[0, \pi / 2]$ between the vectors

$$
\boldsymbol{a}=\sum_{i=1}^{n} a_{i} \boldsymbol{z}_{i}^{\prime}, \quad \boldsymbol{b}=\sum_{i=1}^{n} b_{i} \boldsymbol{z}_{i},
$$

where

$$
\cos \theta=\frac{|(\boldsymbol{a}, \boldsymbol{b})|}{\|\boldsymbol{a}|\||| \boldsymbol{b}\|} .
$$

We remember the following two Lemmas ${ }^{18,50,51,17}$ 
Lemma 1 The squares $\cos ^{2} \theta_{i}$ of the stationary angles between the $n$-planes $Z, Z^{\prime}$ with $\left(\left(Z, Z^{\prime}\right)\right) \neq 0$ are given as the eigenvalues of a matrix $W$ which, for $Z, Z^{\prime} \in \mathcal{V}_{0}$ is

$$
W=\left(\mathbb{1}+Z Z^{+}\right)^{-1}\left(\mathbb{1}+Z Z^{\prime+}\right)\left(\mathbb{1}+Z^{\prime} Z^{\prime+}\right)^{-1}\left(\mathbb{1}+Z^{\prime} Z^{+}\right) .
$$

Lemma 2 Let $\theta$ be the angle defined by the hermitian scalar product in the following equation

$$
\cos \theta\left(Z^{\prime}, Z\right) \equiv \frac{\left|\left(\left(Z^{\prime}, Z\right)\right)\right|}{\left\|Z^{\prime}\right\|\|Z\|}=\frac{\left|\operatorname{det}\left(\mathbb{1}+Z Z^{\prime+}\right)\right|}{\left|\operatorname{det}\left(\mathbb{1}+Z Z^{+}\right)\right|^{1 / 2}\left|\operatorname{det}\left(\mathbb{1}+Z^{\prime} Z^{\prime+}\right)\right|^{1 / 2}},
$$

$d_{c}$ the Cayley distance and $\theta_{1}, \ldots, \theta_{n}$ the stationary angles. Then

$$
\cos \theta\left(Z, Z^{\prime}\right)=\cos d_{c}\left(\iota\left(Z^{\prime}\right), \iota(Z)\right)=\cos \theta_{1} \cdots \cos \theta_{n} .
$$

It can be proved ${ }^{17}$ that the eigenvalues of $\mathrm{W}$ appear also in the expression of the distance on the complex Grassmann manifold (see also Siegel ${ }^{52}$ ).

Note also that if the expression (3.2) of the dependence $Z=Z(B)$ is introduced in the formula of the distance between the points $Z=0$ and $Z \in V_{0}$ on the Grassmann manifold, then

$$
d^{2}=\sum\left|B_{i j}\right|^{2}
$$

The last equation expresses the fact that the parameters $B$ in eq. (2.8) of coherent states are indeed the normal coordinates as it is asserted in Remark 2.

4. Below we present the cut locus and the conjugate locus for $G_{n}\left(\mathbb{C}^{m+n}\right)$.

$\mathrm{O}^{\perp}$ denotes the orthogonal complement of the $n$-plane $\mathbf{O}$ in $\mathbb{C}^{N}$.

Remark 4 (Wong ${ }^{13}$ ) The cut locus of the point $\mathbf{O}$ is given by

$$
\begin{aligned}
\mathbf{C L}_{0} & =\Sigma_{0}=V_{1}^{m}=Z\left(\omega_{1}^{m}\right)=Z(m-1, m, \ldots, m) \\
& =\left\{X \subset G_{n}\left(\mathbb{C}^{n+m}\right) \mid \operatorname{dim}\left(X \cap \mathbf{O}^{\perp}\right) \geq 1\right\} .
\end{aligned}
$$

The cut locus in $G_{n}\left(\mathbb{C}^{m+n}\right)$ is given by those $n$-planes which have at least one of the stationary angles $\pi / 2$ with the plane $\mathbf{O}$.

Proof. An immediate proof can be obtained using the results of Wu referring to the polar divisor $\Sigma_{0}$ on the Grassmann manifold (see Ch. 1 in Ref. 43) and the theorems characterising the canonical (universal, det) bundle on $G_{n}\left(\mathbb{C}^{N}\right)$ (see especially Prop. 3.3 Ch. 7 in Ref. 7), which are particularisations of the representation in Thm. 2.

The following theorem summarize the known facts about the tangent conjugate locus and conjugate locus in $G_{n}\left(\mathbb{C}^{m+n}\right) .{ }^{13,15,17}$ The relevant fact for the present paper is that the conjugate locus can be calculated using Theorem 1. 
Theorem 3 The tangent conjugate locus $C_{0}$ of the point $\mathbf{O} \in G_{n}\left(\mathbb{C}^{m+n}\right)$ is given by

$$
C_{0}=\bigcup_{k, p, q, i} \operatorname{ad} k\left(t_{i} H\right), i=1,2,3 ; 1 \leq p<q \leq r,
$$

where the vector $H \in \mathfrak{a}$ is normalised,

$$
H=\sum_{i=1}^{r} h_{i} D_{i n+i}, h_{i} \in \mathbb{R}, \quad \sum h_{i}^{2}=1 .
$$

The parameters $t_{i}, i=1,2,3$ in eq. (4.26) are

$$
\begin{aligned}
& t_{1}=\frac{\lambda \pi}{\left|h_{p} \pm h_{q}\right|}, \text { multiplicity } 2 \\
& t_{2}=\frac{\lambda \pi}{2\left|h_{p}\right|} \quad, \text { multiplicity } 1 ; \\
& t_{3}=\frac{\lambda \pi}{\left|h_{p}\right|} \quad, \text { multiplicity } 2|m-n| ; \lambda \in \mathbb{Z}^{\star} .
\end{aligned}
$$

The conjugate locus of $\mathbf{O}$ in $G_{n}\left(\mathbb{C}^{m+n}\right)$ is given by the union

$$
\mathbf{C}_{0}=\mathbf{C}_{0}^{W} \cup \mathbf{C}_{0}^{I} \text {. }
$$

The following relations are true

$$
\begin{aligned}
& \mathbf{C}_{0}^{I}=\exp \bigcup_{k, p, q} A d k\left(t_{1} H\right), \\
& \mathbf{C}_{0}^{W}=\exp \bigcup_{k, p} A d k\left(t_{2} H\right),
\end{aligned}
$$

i.e. exponentiating the vectors of the type $t_{1} H$ we get the points of $\mathbf{C}_{0}^{I}$ for which at least two of the stationary angles with $\mathbf{O}$ are equal, while the vectors of the type $t_{2} H$ are sent to the points of $\mathbf{C}_{0}^{W}$ for which at least one of the stationary angles with $\mathbf{O}$ is 0 or $\pi / 2$.

The $\mathbf{C}_{0}^{W}$ part of the conjugate locus is given by the disjoint union

$$
\mathbf{C}_{0}^{W}= \begin{cases}V_{1}^{m} \cup V_{1}^{n}, & n \leq m \\ V_{1}^{m} \cup V_{n-m+1}^{n}, & n>m\end{cases}
$$

where

$$
\begin{aligned}
& V_{1}^{m}= \begin{cases}\mathbb{C P}^{m-1}, & \text { for } n=1 \\
W_{1}^{m} \cup W_{2}^{m} \cup \ldots W_{r-1}^{m} \cup W_{r}^{m}, & 1<n\end{cases} \\
& W_{r}^{m}= \begin{cases}G_{r}\left(\mathbb{C}^{\max (m, n)}\right), & n \neq m, \\
\mathbf{O}^{\perp}, & n=m,\end{cases} \\
& V_{1}^{n}= \begin{cases}W_{1}^{n} \cup \ldots \cup W_{r-1}^{n} \cup \mathbf{O}, & 1<n \leq m, \\
\mathbf{O}, & n=1,\end{cases} \\
& V_{n-m+1}^{n}=W_{n-m+1}^{n} \cup W_{n-m+2}^{n} \cup \ldots \cup W_{n-1}^{n} \cup \mathbf{O}, n>m .
\end{aligned}
$$


Sketch of the Proof. The tangent conjugate locus $C_{0}$ for $G_{n}\left(\mathbb{C}^{m+n}\right)$ in the case $n \leq m$ was obtained by Sakai. ${ }^{15}$ Sakai has observed that Wong's result on the conjugate locus in the manifold is incomplete, i.e. $\mathbf{C}_{0}^{W} \subset \mathbf{C}_{0}$ but $\mathbf{C}_{0}^{W} \varsubsetneqq \mathbf{C}_{0}=\exp C_{0}$. The proof of Sakai consists in solving the eigenvalue equation $R\left(X, Y^{i}\right) X=e_{i} Y^{i}$ which appears when solving the Jacobi equation, where the curvature for the symmetric space $X_{c}=G_{c} / K$ at $o$ is simply $R(X, Y) Z=[[X, Y], Z], X, Y, Z \in \mathfrak{m}_{c}$. Then $q=\operatorname{Exp}_{0} t X$ is conjugate to $o$ if $t=\pi \lambda / \sqrt{e_{i}}, \lambda \in \mathbb{Z}^{\star} \equiv \mathbb{Z} \backslash\{0\}$.

Above $\mathfrak{a}$ is the Cartan subalgebra of the symmetric pair $(S U(n+m), S(U(n) \times$ $U(m)))^{12,15,17}$ consisting of vectors of the form (4.27) where $r$ is the symmetric rank of $X_{c}$ (and $X_{n}$ ) and we use the notation $D_{i j}=E_{i j}-E_{j i}, i, j=1, \ldots, N$. $E_{i j}$ is the matrix with entry 1 on line $i$ and column $j$ and 0 otherwise. The results in the complex Grassmann manifold are obtained farther using the exponential map given by eq. (3.2).

The same result on the calculation of the tangent conjugate locus can be obtained ${ }^{17}$ using Prop. 3.1 p. 294 in the book of Helgason. ${ }^{12}$ This Proposition asserts that $H \in \mathfrak{a}$ is conjugate with $o$ iff $\alpha(H) \in i \pi \mathbb{Z}^{\star}$ for some root $\alpha$ which do not vanishing identically on $\mathfrak{a}$. The eigenvalues of the equation $[H, X]=\lambda X, \forall H \in \mathfrak{a}$, lead ${ }^{17}$ to the values given in equation (4.26) for the parameters $t_{1}-t_{3}$.

The direct proof ${ }^{17}$ in the Grassmann manifold uses in Theorem 1 the dependence $Z=Z(B)$ furnished by eq. (3.2) which gives the geodesics on $G_{n}\left(\mathbb{C}^{n+m}\right)$ and the Jordan's stationary angles between two $n$-planes. The stationary angles between two $n$-planes are given by Lemma 1 and appear in the relation given by Lemma 2 .

The proof ${ }^{17}$ is done in four steps. a) Firstly, a diagonalization of the $n \times m$ matrix $Z$ is performed. b) Secondly, the Jacobian of a transformation of complex dimension one is computed. c) The cut locus is reobtained and his contribution to the conjugate locus is taken into account. d) The nonzero angles are counted using the following property of the stationary angles: if the $n^{\prime}(n)$-plane (resp. $Z_{n}$ ) are such that $Z_{n^{\prime}}^{\prime} \cap Z_{n}=Z^{\prime \prime} n^{\prime \prime}$, than $n^{\prime}-n^{\prime \prime}$ angles of $Z_{n^{\prime}}^{\prime}$ and $Z_{n}$ are different from 0 and $n "$ are 0 .

\section{CONCLUSION AND DISCUSSION}

In this paper it was shown that for a certain class of homogeneous manifolds which include the symmetric ones there is a relationship between geodesics and coherent states. The starting point ${ }^{10}$ of the present investigation, contained in Remark 1, is the observation that for symmetric spaces, if one expresses the parameters $Z$ in eq. (2.3) as a function of the parameters $B$ in eq. (2.8), both characterising the coherent states, explicit local formulas for the geodesic exponential map are obtained. For Hermitian symmetric spaces the dependence $Z=Z(B)$ can be found using the Harish-Chandra decomposition or the so called Baker-Campbell-Hausdorff formulas. ${ }^{10}$ Thus Theorem 1 permits a calculation of the conjugate locus in $G_{n}\left(\mathbb{C}^{m+n}\right)$. However, the explicit form of the conjugate locus in $G_{n}\left(\mathbb{C}^{m+n}\right)$ is not completely known. ${ }^{17}$ The part of the conjugate locus $\mathbf{C}_{0}^{W}$ determined by Wong is expressible as Schubert varieties, ${ }^{14}$ while the rest ${ }^{15}$ $\mathbf{C}_{0}^{I}$ can be characterised ${ }^{17}$ as the subset of points of $G_{n}\left(\mathbb{C}^{m+n}\right)$ which have at least two of the stationary angles with the fixed $n$-plane $\mathbf{O}$ equal. $\mathbf{C}_{0}^{I}$ contains as subset the maximal set of mutually isoclinic ${ }^{53}$ subspaces of the Grassmann manifold, which are isoclinic spheres, ${ }^{53,54}$ with dimension given by the solution of the Hurwitz ${ }^{55}$ problem. This part referring to the explicit calculation of the conjugate locus on $G_{n}\left(\mathbb{C}^{m+n}\right)$ was 
only briefly included in Sec. IV, the full details being presented elsewhere. ${ }^{17}$

The main remark of this paper contained in Theorem 2, the equality (1.1), is a simple consequence of the fact that any manifold is the disjoint union of a maximal normal neighbourhood $\mathcal{V}_{0}$ of a point 0 and the cut locus $\mathbf{C L}_{0}$. It would be interesting to find a geometrical description of the polar divisor for manifolds which are not characterised by condition $B$ ). On the other side, the problem to find explicitly the cut locus on nonsymmetric spaces is a difficult one. ${ }^{16}$ Also it was proved that for homogeneous manifolds verifying the condition $B$ ) and admitting an embedding in an adequate projective Hilbert space a necessary and sufficient condition that a point to belong to the cut locus of another point is that the Cayley distance between the images of the points through the embedding to be $\pi / 2$. This category of manifolds includes all the coherent states manifolds which admit prequantization. ${ }^{19}$

\section{ACKNOWLEDGEMENTS}

The author expresses his gratitude to Prof. Anne Boutet de Monvel and CNRS for the opportunity to work during the summers 1993 and 1994 in the Équipe de Physique Mathématique et Géométrie at the Institute de Mathématique de l'Université Paris 7 Denis Diderot. The constant interest of Professor L. Boutet de Monvel is kindly acknowledged. Discussions during the XIII and XIV Workshops on Geometrical Methods in Physics at Białowieża, especially with Professors D. Simms, M. Cahen, A. M. Perelomov and J. Klauder are acknowledged. The author also expresses his thanks to Professors K. Teleman, S. Kobayashi, M. Berger, T. Sakai and Th. Hangan for suggestions.

${ }^{1}$ Coherent States, edited by J. R. Klauder and B. S. Skagerstam (Word Scientific, Singapore, 1985).

${ }^{2}$ S. Berceanu, "From quantum mechanics to classical mechanics and back, via coherent states", in Quantization and Infinite-Dimensional Systems (Plenum, New York, 1994), p. 155 .

${ }^{3}$ A. M. Perelomov, "Coherent states for arbitrary Lie groups", Commun. Math. Phys. 26, 222 (1972).

${ }^{4}$ J. R. Rawnsley, "Coherent states and Kähler manifolds", Quart. J. Math. Oxford 28, 403 (1977).

${ }^{5}$ B. Kostant, "Quantization and unitary representations", in Lecture Notes in Mathematics, Vol. 170, edited by C. T. Taam (Springer-Verlag, Berlin 1970), p. 87.

${ }^{6}$ S. S. Chern, Complex Manifolds without Potential Theory (Van Nostrand, Princeton, 1967).

${ }^{7}$ D. Husemoller, Fibre Bundles (Mc Graw-Hill, New York 1966).

${ }^{8}$ F. Hirzebruch, Topological Methods in Algebraic Geometry (Springer-Verlag, Berlin, 1966).

${ }^{9}$ V. Ceausescu and A. Gheorghe, "Classical limit and quantization of Hamiltonian systems", in Symmetries and Semiclassical Features of Nuclear Dynamics, Lecture Notes in Physics, Vol. 279, edited by A. A. Raduta (Springer-Verlag, Berlin, 1987), p. 69.

${ }^{10}$ S. Berceanu and L. Boutet de Monvel, "Linear dynamical systems, coherent state manifolds, 
flows and matrix Riccati equation", J. Math. Phys. 34, 2353 (1993).

${ }^{11}$ S. Kobayashi and K. Nomizu, Foundations of Differential Geometry, Vol. 11 (Interscience, New York, 1969).

${ }^{12}$ S. Helgason, Differential Geometry, Lie groups and Symmetric Spaces (Academic, New York, 1978).

${ }^{13}$ Y. -C. Wong, "Differential Geometry of Grassmann manifolds", Proc. Nat. Acad. Sci. U.S.A. 57, 589 (1967).

${ }^{14}$ Y. -C. Wong, "Conjugate loci in Grassmann manifold", Bull. Am. Math. Soc. 74, 240 (1968).

15 T. Sakai, "On cut loci on compact symmetric spaces", Hokkaido Math. J. 6, 136 (1977).

${ }^{16}$ S. Kobayashi, "On conjugate and cut loci", in Global Differential Geometry, M.A.A. Studies in Mathematics, Vol. 27, S. S. Chern editor (1989), p. 140.

${ }^{17} \mathrm{~S}$. Berceanu, "On the Geometry of complex Grassmann manifold, its noncompact dual and coherent states", preprint Bucharest, Institute of Atomic Physics FT-409-1995, September.

${ }^{18}$ C. Jordan, "Essai sur la Géométrie à n dimensions", Bull. Soc. Math. France t. 1ll, 103 (1875).

${ }^{19}$ S. Berceanu, "The coherent states: old geometrical methods in new quantum clothes", poster presented at the XI ${ }^{\text {ème }}$ Congrès International de Physique Mathématique, Paris, 18-23 Juillet (1994); preprint Bucharest, Institute of Atomic Physics, FT-398-1994 and preprint Universität Bielefeld BiBoS Nr. 664/11/94.

20 S. Berceanu, "Coherent states and global Differential Geometry", talk presented at the XIII Workshop on Geometrical Methods in Physics at Białowieża, Poland, July 9-15 (1994), to appear in the Proceeding of the Workshop Quantisation, coherent states, and complex structures, edited by J. P. Antoine, S. T. Ali, W. Lisiecki, I. Mladenov and A. Odzijewicz, Plenum (1995).

${ }^{21}$ V. Guillemin and S. Sternberg, Symplectic Techniques in Physics (Cambridge U. P., London, 1984).

22 A. Odzijewicz, "Coherent states and geometric quantization", Commun. Math. Phys. 150, 85 (1992).

${ }^{23}$ A. A. Kirillov, Elements of Theory of Representations (Springer-Verlag, New York, 1976).

${ }^{24}$ E. Onofri, "On quantization theory for homogeneous Kähler spaces", preprint Parma IFPRT-038 (1974).

${ }^{25}$ H. Woodhouse, Geometric Quantization (Oxford U. P., Oxford, 1980).

${ }^{26}$ A. W. Knapp, Representation Theory of Semisimple Lie Groups (Princeton, NJ, 1986).

${ }^{27}$ M. Cahen, S. Gutt and J. Rawnsley, "Quantization on Kähler manifolds", ll, Trans. Math. Soc. 337, 73 (1993)

${ }^{28}$ B. Shiffman and A. J. Sommese, Vanishing Theorems on Complex Manifolds, Progress in Mathematics, Vol. 56 (Birkhäuser, Boston, 1985).

${ }^{29}$ P. Griffith and J. Harris, Principles of Algebraic Geometry (Wiley, New York, 1978).

${ }^{30} \mathrm{~S}$. Berceanu and A. Gheorghe, "On the construction of perfect Morse functions on compact manifolds of coherent states", J. Math. Phys. 28, 2899 (1987).

${ }^{31}$ S. Kobayashi, "On the Geometry of bounded domains", Trans. Amer. Math. Soc. 92, 267 (1959).

32 W. Lisiecki, "A classification of coherent state representations of unimodular Lie groups", Bull. Amer. Math. Soc. 25, 37 (1991); "Coherent state representations. A survey", to appear in Math. Rep. (1995).

${ }^{33}$ R. Crittenden, "Minimum and conjugate points in symmetric spaces", Canad. J. Math. 14, 320 (1962).

${ }^{34}$ F. W. Warner, "The conjugate locus of a Riemannian manifold", Amer. J. Math. 87, 575 (1965). 
35 A. D. Weinstein, "The cut locus and conjugate locus of a Riemannian manifold", Ann. of Math. 87, 29 (1968).

${ }^{36}$ Y. -C. Wong, "A class of Schubert varieties", J. Diff. Geom. 4, 37 (1970).

37 T. Sakai, "The manifold of the Lagrangean subspaces of a symplectic vector space", J. Diff. Geom. 12, 555 (1977).

38 T. Sakai, "On the structure of cut loci in compact riemannian symmetric spaces", Math. Ann. 235129 (1978).

${ }^{39}$ M. Takeuchi, "On conjugate loci and cut loci of compact symmetric spaces " I, II, Tsukuba Math. J. 2, 35 (1977); 3, 1 (1979).

${ }^{40}$ A. Thimm, "Integrable geodesic flows on homogeneous spaces", Ergod. Theory Dyn. Syst. 1, 495 (1981).

${ }^{41}$ O. Kowalski, Generalised Symmetric Spaces, Lecture Notes in Mathematics Vol. 805 (Springer-Verlag, Berlin, 1980).

42 R. Montgomery, "Isoholonomic problems and some applications", Comm. Math. Phys. 128, 565 (1990).

${ }^{43}$ H. H. Wu, The Equidistribution Theory of Holomorphic Curves, Annals of Maths. Studies Vol. 164 (Princeton, New Jersey, 1970).

${ }^{44}$ A. Cayley, "A sixth memoir upon quantics", Phil. Trans. Royal. Soc. London 149, 61 (1859).

${ }^{45}$ F. P. Gantmacher, Teoria Matritz (Nauka, Moskwa, 1966).

${ }^{46} \mathrm{~S}$. Berceanu and A. Gheorghe, "Perfect Morse functions on manifold of Slater determinants", Rev. Roum. Phys. 34, 125 (1989).

${ }^{47}$ L. C. Pontrjagin, "Charakteristiceskie tzikly differentziruemyh mnogobrazia", Mat. sb. 21, 233 (1947).

${ }^{48}$ J. Milnor and J. D. Stasheff, Characteristic Classes, Annals of Maths. Studies Vol. 76 (Princeton, New Jersey, 1974).

49 C. Ehresman, "Sur la topologie de certain espaces homogenès", Ann. Math. 35, 396 (1934).

${ }^{50}$ B. Rosenfel'd, "Vnnutrenyaya geometriya mnojestva m-mernyh ploskastei n-mernova ellipticeskovo prostranstva", Izv. Akad. Nauk. SSSR, ser. mat. 5, 353 (1941).

${ }^{51}$ B. Rosenfel'd, Mnogomernye Prostranstva (Nauka, Moskwa, 1966); Neevklidovy Pronstranstva (Nauka, Moskwa, 1969).

${ }^{52}$ C. L. Siegel, Symplectic Geometry (Academic Press, New York, 1964).

${ }^{53}$ Y. -C. Wong, "Isoclinic $n$-planes in Euclidean $2 n$-space, Clifford parallels in elliptic $(2 n-1)$ space, and the Hurwitz matrix equations", Mem. Amer. Math. Soc. 41 (1961).

${ }^{54}$ J. A. Wolf, "Geodesic spheres in Grassmann manifolds", Ilin. J. Math. 7, 425 (1963); "Elliptic spaces in Grassmann manifolds", Ilin. J. Math. 7, 447 (1963).

55 A. Hurwitz, "Über die Komposition der quadratischen Formen von beliebig vielen Variablen", Nach. v. der Ges. der. Wiss., Götingen (Math. Phys. K1) 309, (1898); reprinted in Math. Werke Bd. 2, p. 641. 